\title{
8
}
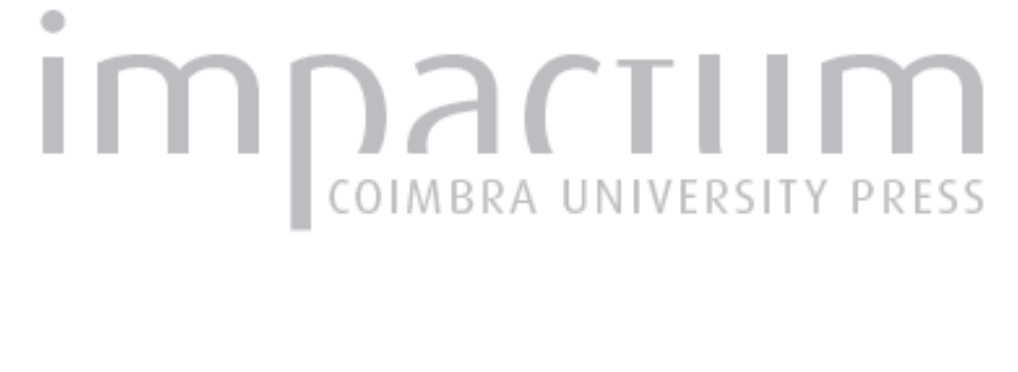

\section{Instituciones en chile para la defensa contra incendios de interfaz urbano-forestal}
Autor(es):
S., Miguel Castillo

Publicado por:

Associação Portuguesa de Riscos, Prevenção e Segurança; Imprensa

URL

persistente: da Universidade de Coimbra

DOI:

URI:http://hdl.handle.net/10316.2/39796

Accessed : $\quad$ 26-Apr-2023 04:52:09

DOI:https://doi.org/10.14195/1647-7723_23_18

A navegação consulta e descarregamento dos títulos inseridos nas Bibliotecas Digitais UC Digitalis, UC Pombalina e UC Impactum, pressupõem a aceitação plena e sem reservas dos Termos e Condições de Uso destas Bibliotecas Digitais, disponíveis em https://digitalis.uc.pt/pt-pt/termos.

Conforme exposto nos referidos Termos e Condições de Uso, o descarregamento de títulos de acesso restrito requer uma licença válida de autorização devendo o utilizador aceder ao(s) documento(s) a partir de um endereço de IP da instituição detentora da supramencionada licença.

Ao utilizador é apenas permitido o descarregamento para uso pessoal, pelo que o emprego do(s) título(s) descarregado(s) para outro fim, designadamente comercial, carece de autorização do respetivo autor ou editor da obra.

Na medida em que todas as obras da UC Digitalis se encontram protegidas pelo Código do Direito de Autor e Direitos Conexos e demais legislação aplicável, toda a cópia, parcial ou total, deste documento, nos casos em que é legalmente admitida, deverá conter ou fazer-se acompanhar por este aviso.

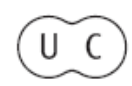




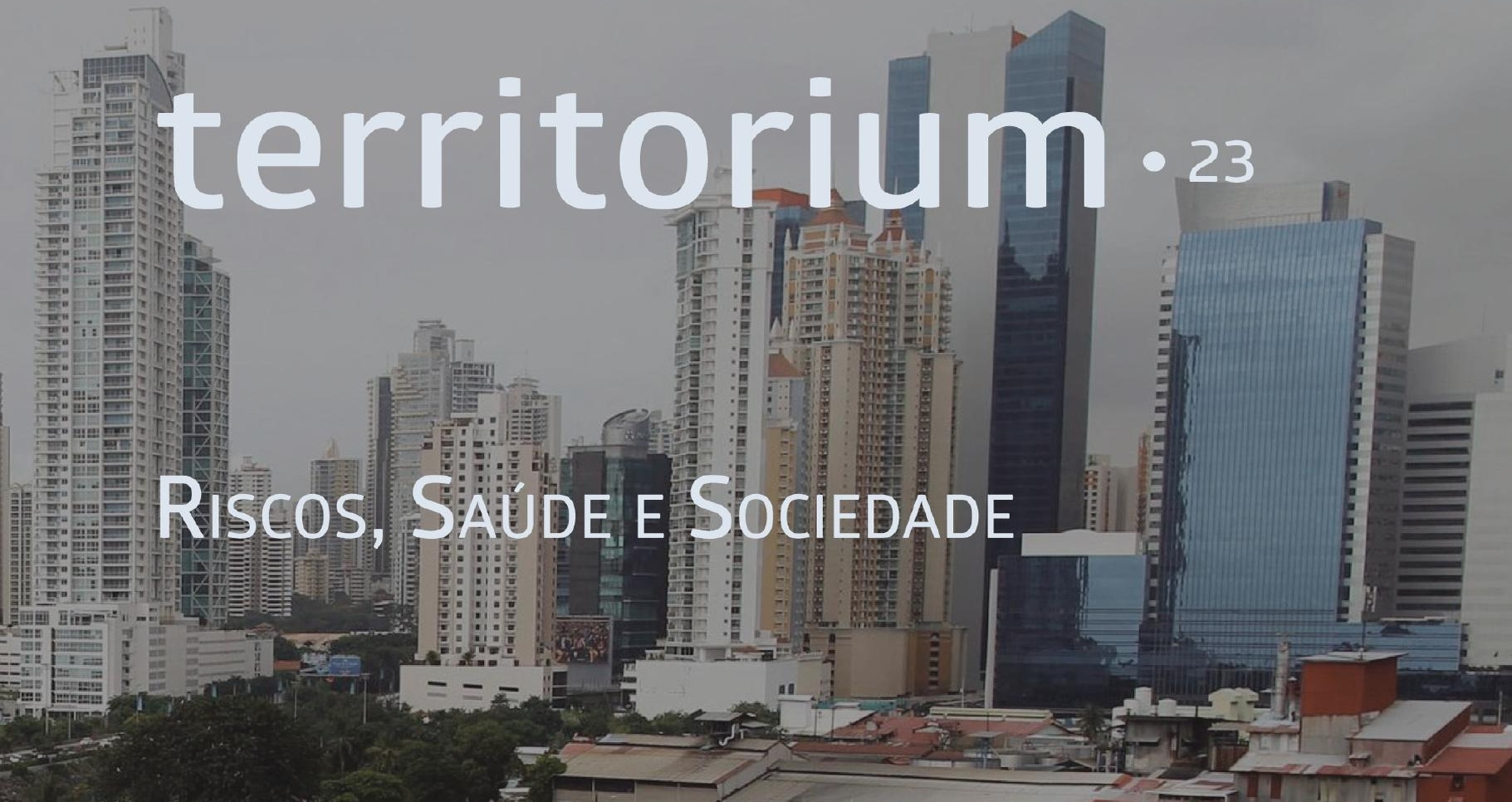

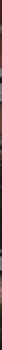

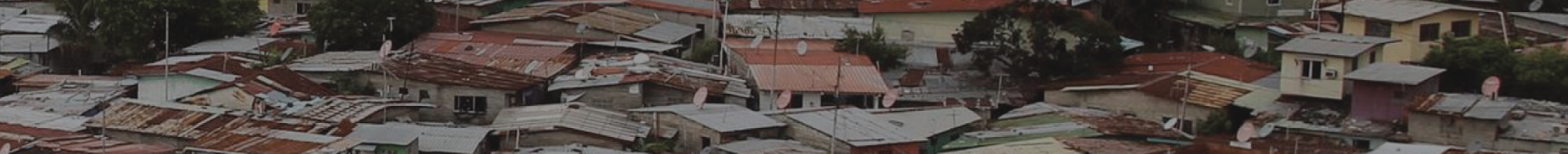

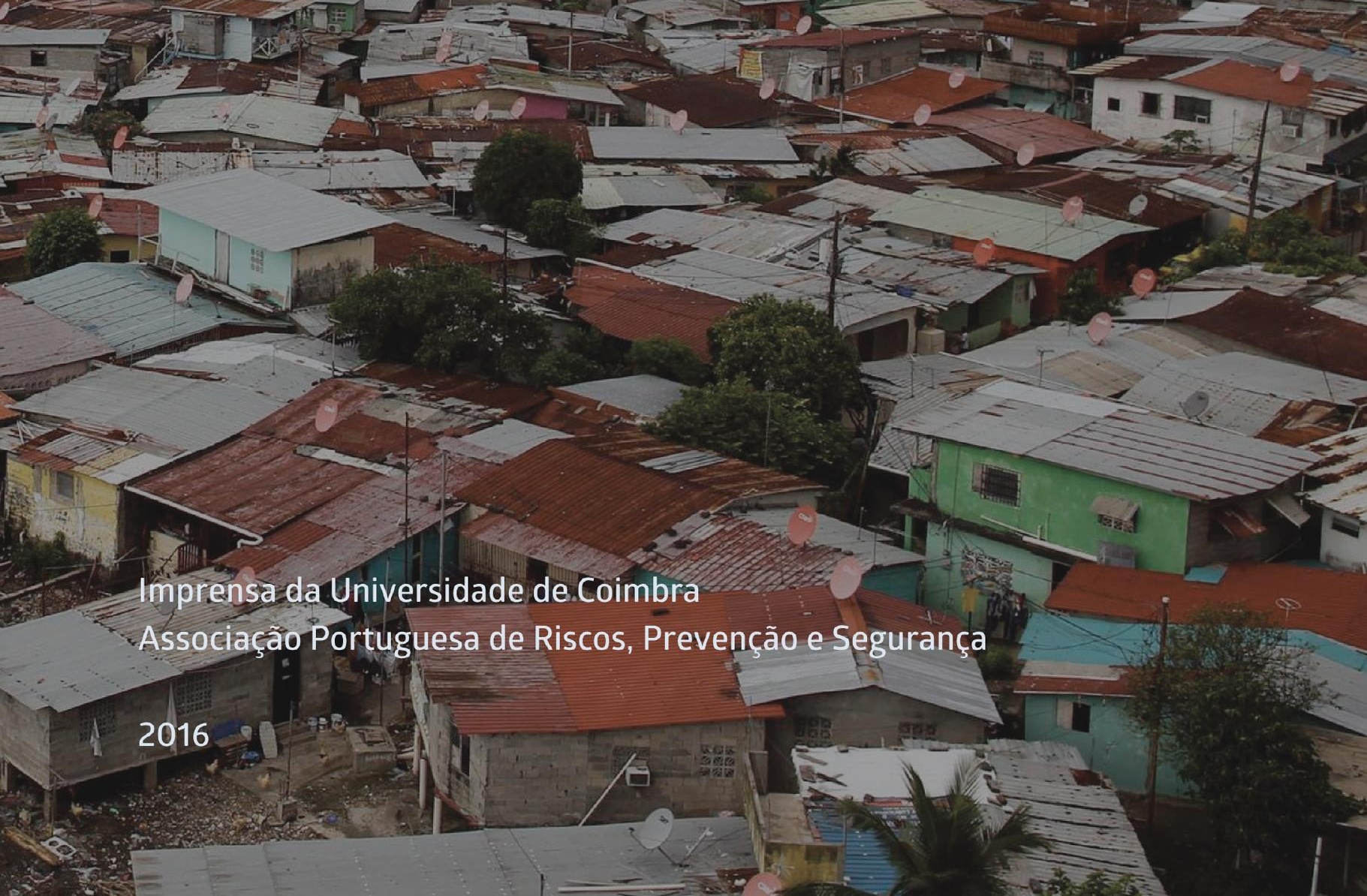




\section{INSTITUCIONES EN CHILE PARA LA DEFENSA CONTRA INCENDIOS DE INTERFAZ URBANO-FORESTAL*}

INSTITUTIONS IN CHILE FOR FIRE PROTECTION OF FOREST-URBAN INTERFACE

Miguel Castillo S.

Laboratorio de Incendios Forestales. Universidad de Chile migcasti@uchile.cl

\section{RESUMEN}

Chile ha tenido un notable aumento en el riesgo de incendios de interfaz urbano-forestal. También se ha constatado un elevado nivel de daños producidos por la propagación del fuego en estas áreas. Ello se ha debido, entre otros factores, por las prolongadas sequías asociadas a condiciones meteorológicas muy desfavorables, el aumento de la intencionalidad en la iniciación de incendios, debilidades en las campañas de prevención y manejo de la vegetación combustible, y también por deficiencias en las políticas de gestión del territorio. En este artículo se describen las principales instituciones que se encargan de proteger al país frente a este problema.

Palabras clave: Gestión del riesgo, peligro de incendios, interfaz urbano-forestal, causas de incendios, planificación territorial.

\section{ABSTRACT}

Chile has seen a considerable increase in the fire risk in the forest-urban interface. Furthermore, there has been a high level of damage resulting from fire propagation in these areas. This is due, among other factors, to prolonged droughts associated with very unfavorable weather conditions, an increase in arson, shortcomings in prevention campaigns and in fire propellant vegetation handling and also due to deficiencies in land management policies. This article describes the main institutions responsible for protecting the country against this problem.

Keywords: Risk management, fire danger, forest-urban interface, causes of fire, territorial planning.

Introducción

Chile presenta un desarrollo en materia de protección contra incendios forestales que es reconocido a nivel sudamericano, por cuanto basa su desarrollo formal desde el año 1967 con la implementación del primer programa de defensa contra incendios, fortaleciendo los planes y programas de prevención y combate, tanto en el ámbito privado como en las instituciones del Estado.

La actual demanda en bienes y servicios derivados de la explotación de los recursos naturales renovables - entre ellos los bosques - junto a la creciente presión por ocupación del suelo en espacios silvestres, ha tenido como consecuencia un aumento sostenido en los niveles de riesgo de incendios, los cuales han aumentado su grado de severidad porque además se han ido sucediendo paralelamente a otros factores que indudablemente han aportado al escenario de ocurrencia: la variable climática cada vez más severa, el incremento de la intencionalidad en la iniciación de focos de fuego, en la acumulación permanente de energía no liberada por la inadecuada o insuficiente práctica preventiva en el manejo de la vegetación combustible, en el aumento del efecto borde de caminos hacia espacios silvestres, y en la instalación de viviendas en sectores no preparados para la este uso.

Por lo anterior, las instituciones de defensa contra incendios forestales en Chile, han tenido la necesidad de ir adaptándose al contexto histórico de la evolución de este problema en el país, estableciendo políticas y formas de trabajo coordinado que han permitido en forma parcial, mitigar el riesgo y peligro potencial de la propagación del fuego, junto con disponer de mecanismos para dar respuesta oportuna a eventos de multiocurrencia, algo que sucede normalmente en época de verano en Chile Mediterráneo.

\section{El estudio de la interfaz urbano-forestal en Chile}

En un contexto histórico, los técnicos planificadores y tomadores de decisiones políticas, consideraban solo

\footnotetext{
* O texto desta nota foi submetida em 03-01-2015, sujeita a revisão por pares a 30-04-2015 e aceite para publicação em 01-07-2015. Esta nota é parte integrante da Revista Territorium, n. ${ }^{\circ} 23,2016,{ }^{\circ}$ RIscos, ISSN: 0872-8941.
} 
dos dimensiones del territorio, la rural y la urbana. Actualmente se sabe que existe una tercera dimensión o aspecto a analizar, y que corresponde a una faja o área intermedia denominada zona de interfaz (urbano-rural o urbano-forestal, según sea el caso), donde se combinan usos residenciales, productivos y recreativos, en un entorno con características rurales y con una vegetación forestal a su alrededor. Stewart et al., 2007 consideran como componentes esenciales de la interfaz, la presencia humana, las características de la vegetación circundante y la relación de distancia hacia la interfaz (definida por los autores como buffer). Para analizar el problema de los incendios forestales en esta urbanización dispersa, apareció en los países anglosajones a principios de los 90 un nuevo concepto denominado wildland-urban interface, WUI. La definición de WUI fue desarrollada inicialmente en Estados Unidos con el fin de identificar aquellas zonas con un alto riesgo de incendios.

La destrucción de la vegetación y casas colindantes producto de la acción descontrolada del fuego es actualmente un tema de extrema preocupación por parte de las autoridades relacionadas a la prevención y control de incendios forestales en prácticamente todos los países de ecosistemas mediterráneos y Norteamérica. En la mayoría de los casos se verifica un aumento sostenido de los incendios en zonas de interfaz, y que no dan señales de disminuir en número y gravedad de los mismos, aún cuando se ha visto el notable aumento en presupuesto destinado a las operaciones de prevención y combate de incendios. Es una realidad que se ve prácticamente todos los años en España, Grecia, Portugal, Italia, Estados Unidos, y particularmente grave en Chile en la temporada de incendios 2013-2014.

Al respecto, Chile es un país permanentemente afectado por incendios forestales, y en los últimos 15 años con mayor énfasis en incendios de interfaz. Si bien es cierto que la acción y aplicación del fuego puede ser considerada como una muy buena herramienta como apoyo a la ejecución de labores agrícolas y forestales, se ha visto con mucha preocupación el aumento sostenido de la intencionalidad e irresponsabilidad en el uso del fuego, lo que ha llevado a que las cifras de ocurrencia y superficie dañada hayan tenido un notable ascenso, y lo más grave, en un aumento de la severidad y gravedad por los efectos que el fuego ha producido en reiteradas ocasiones en áreas donde ya se ha visto que los daños potenciales son enormes. Como se indicaba anteriormente, el concepto de incendios en bosque ha cambiado a incendios de interfaz urbano-forestal, como un reflejo de las condiciones cambiantes de un territorio bajo permanente competencia por el uso del suelo.

Normalmente en esta interfaz se genera una variedad de situaciones intermedias que permiten la conexión entre paisajes. En estas áreas es común encontrar incendios, cuya causa es principalmente por actividad humana. De hecho, muchos eventos de fuegos que se producen en esta área de contacto se derivan de distintas actividades que se practican, tales como extracción de material vegetal, depósito de desperdicios, o recreación, entre otros factores. En forma adicional, la urbanización y el incremento de la densidad de población traen como consecuencia la fragmentación de áreas aledañas, con el consiguiente riesgo de degradación de espacios naturales (Zhai et al., 2003). De esta manera, cuando la población crece, comienza la presión por la utilización del espacio y sus recursos asociados. Generalmente esto se expresa en la reconversión de terrenos agrestes por tierras productivas o simplemente para construcción de nuevos espacios urbanos, normalmente en áreas que no han sido estudiadas a fondo como para ser declaradas para uso habitacional. Justamente lo anterior se transforma en un conflicto de tipo ambiental y social, porque en muchas de estas áreas la presión del hombre ocasiona graves incendios de interfaz, dejando además otras consecuencias importantes, como es la fragmentación de paisajes y deterioro de la calidad de vida. Por ejemplo, en Estados Unidos, más de 900 viviendas son destruidas anualmente en promedio, producto de incendios generados en áreas de interfaz, de acuerdo a estadísticas reportadas a partir de 1990.

\section{La interfaz y su relación con los incendios forestales}

Referente a las zonas de interfaz en la Región de Valparaíso, Castillo (2013) explica que uno de los temas más complicados en el desarrollo explosivo de un incendio, es cuán rápido avanza el fuego por los cerros y casas. Valores referenciales medidos en condiciones reales señalan velocidades medias en un rango de 40 a 300 metros $^{2}$ /minuto. Sin embargo en un comportamiento extremo del fuego se ha visto que esta velocidad puede superar en algunos casos los 600 metros $^{2} /$ minuto, lo que equivale a algo más de 3,2 hectáreas/hora. Un escenario aún más extremo fue el que se presentó en abril de 2014 en Valparaíso. En este gran incendio, según mediciones de campo y vuelos aéreos, el fuego llegó a avanzar hasta $1.300 \mathrm{~m}^{2} /$ minuto, lo que equivale aproximadamente a 8 hectáreas/hora, cifras sin precedentes y que escapan a toda escala de medición. En el caso más dramático, se llegó a determinar que en este mismo incendio en un instante puntual el fuego logró avanzar hasta 156 hectáreas/hora, correspondiendo a un incremento exponencial de intensidad calórica, que arrasó extensas superficies en escasos minutos.

En relación al material de construcción de las casas amenazadas por incendios, el comportamiento del fuego en estructuras y construcciones difiere ostensiblemente del que se presenta en espacios abiertos afectando a vegetación combustible. En estas áreas de contacto, 
la velocidad de propagación del fuego y los montos calóricos generados en la combustión de materiales ligeros estructurales ha sido estudiada mediante modelos matemáticos (Porterie et al., 2007) que demuestran que la expansión puede llegar a generar torbellinos de fuego favorecidos por la formación de ciclones de aire caliente que interactúan con los gases incandescentes al interior de estructuras aireadas (Brink et al., 2000). Pyne et al. (1996) han reportado tiempos de residencia de las llamas muy superiores a los que se presentan habitualmente en la combustión de vegetación arbórea o arbustiva en espacios abiertos. Esto hace que las posibilidades de rápida propagación del fuego en construcciones habitacionales sean aún mayores si la densidad de edificaciones combustibles es también alta (Cohen, 1995). En muchos casos, residentes que habitan en zonas de alto riesgo de incendios forestales, prefieren aceptar la presencia del fuego y organizarse en el combate del mismo, más que tomar las precauciones necesarias para minimizar el riesgo en la ignición y propagación.

El peligro de la propagación del fuego depende de distintos factores. Los principales son: el viento (velocidad y dirección), la topografía (pendiente del terreno), y la vegetación combustible. Los dos primeros factores son prácticamente imposibles de controlar en un plan de protección. En cambio la vegetación sí puede ser controlada mediante un adecuado manejo forestal en zonas aledañas a quebradas y centros densamente poblados, lo que indudablemente posibilita la reducción del peligro frente al comportamiento del fuego. El manejo contempla actividades tales como la poda, disminución de la carga de combustible disponible en los estratos horizontal y vertical, limpieza de basurales en fondos de laderas, aumento de clareos y reforzamiento de vías de acceso para el eventual control del fuego (Beebe y Omi, 1993; Julio, 2014). Sin embargo, estas prácticas en muchos casos no se hacen, en otros casos mal ejecutadas, o en grado insuficiente respecto a la demanda en protección, especialmente en aquellos lugares en donde la presencia de altas pendientes y vientos locales intensos, podrían desencadenar grandes incendios de rápida propagación (Beebe y Omi, 1993). En parte, esta fue una de las principales razones que explican la violenta propagación del fuego en Valparaíso durante abril de 2014. En pocas horas ardieron más de 1.000 de casas atrapadas entre una densa vegetación combustible, y que en muchas áreas no fue adecuadamente intervenida con fines de prevención.

Para tratar de mitigar y prevenir este tipo de tragedias, en Estados Unidos se crearon estándares nacionales aplicados para disminuir las condiciones de riesgo en la WUI son regulados por la NFPA (Nacional FIRE Protection Association), y programas de colaboración asociados. Estos estándares tocan aspectos tales como organización de cuerpo de voluntarios, acciones programadas de la comunidad en zonas de riesgo de incendios, ordenanzas municipales de emergencia en situaciones coyunturales y apoyo estatal mediante activación de mecanismos de ayuda directa (Kundell, et al., 2002). De esta manera, ante la gravedad que reviste el problema de los incendios forestales en esta interfaz, en Estados Unidos se han ejecutado diversos proyectos de educación coordinados por la NWCG (Nacional Wildfire Coordinating Comité), mediante la ejecución de talleres participativos con comunidades locales respecto al manejo de vegetación combustible, estrategias de reacción frente a emergencias y comportamiento colectivo, todos estos aspectos, como medidas prácticas para lograr disminuir el peligro de incendios en la interfaz (Kundell, et al., 2002). En Chile, se han ejecutado acciones pero más bien de carácter local, y que no han tenido la continuidad necesaria ni una adecuada evaluación en el tiempo, como para disponer formalmente con un programa integrado de protección contra incendios.

Es común distinguir mezclas en esta zona de interfaz. Davis (1990), y Radeloff et al., (2005), distinguen por ejemplo, cinco categorías o mezclas de situaciones en esta zona WUI o interfaz: El anillo o área WUI propiamente tal, conformada por segmentosclaramente demarcados que separan estructuras estrechamente unidas y consolidadas de otras provisorias o de menor elaboración, un área siguiente (denominada intermix), compuesta por estructuras dispersas, pero siempre manteniendo continuidad de combustible; se distingue además un área de oclusión, compuesta por islas o fragmentos cohesionados de combustibles, frecuentemente pequeñas construcciones aisladas de material ligero; posteriormente aparece un área de borde en donde coexisten asentamientos poblados y enclaves de vegetación combustible en sectores con topografía variable; finalmente se encuentra la vegetación propiamente tal, rodeando en muchos casos, las áreas anteriormente descritas. En el caso de Valparaíso, Rodríguez et al., (2010), realizaron un minucioso y detallado catastro de viviendas en áreas de interfaz, determinando cuatro categorías en función de los materiales de construcción y de la localización de las mismas en los cerros. Las propiedades estructurales y características propias de la localización de las viviendas permitió asociarlas al factor de peligro de incendios. Como resultado, se obtuvo una evaluación económica de tipo preliminar, y también una estimación del número de casas localizadas en interfaz que potencialmente podrían ser afectadas por futuros incendios forestales.

Un tema muy asociado a lo anterior, es la protección civil y estrategias de evacuación frente a emergencias. El primer aspecto a considerar en una estrategia de 
protección contra incendios en áreas de interfaz, es resguardar la seguridad de la población civil. Es el rol primordial del estado garantizar que frente a cualquier tipo de catástrofe, existan a lo menos los mecanismos básicos necesarios para organizar la evacuación, combate y distribución de los recursos necesarios para enfrentar el problema de los incendios en zonas de interfaz, especialmente cuando la propagación del fuego adquiere características de alta conflictividad. En este sentido lo que se ha hecho tradicionalmente en Chile y en otros países de clima mediterráneo es tratar de disponer de la mayor cantidad de recursos financieros disponibles para la prevención y el combate. No obstante se sabe que históricamente siempre estos recursos son insuficientes, estimándose que para el caso de Chile, y en particular en la Región de Valparaíso, debería ser a lo menos el doble de lo actual, como para disponer de un estándar mínimo de protección. No obstante se ha visto que aún cuando se percibe un sostenido incremento en dinero para enfrentar este problema, los incendios están aumentando en número y en severidad de los mismos.

Normalmente los mecanismos tradicionales comienzan por el análisis geográfico y financiero de los recursos disponibles para el combate, olvidando en muchos casos las estrategias de prevención, entre ellas, el adecuado manejo de la vegetación combustible. Cuando las condiciones meteorológicas, topográficas y estructurales de las zonas de riesgo son favorables para la iniciación de incendios, lo que normalmente se aplica es la definición de categorías de alerta, lo que implica entonces la ejecución de distintos procedimientos para ejecutar en terreno las acciones de control de una situación de incendios en conflicto.

No obstante lo anterior, hasta el momento existe en Chile el grave problema de no contar con una evaluación y diagnóstico objetivo, que permita respaldar de mejor forma un plan integrado de protección contra incendios en zonas de interfaz, con medidas a la vez que sean factibles de aplicar, y oportunas. Prueba de ello han sido las constantes tragedias que han ocurrido en Valparaíso y que seguirán ocurriendo si no se cuenta con un adecuado mecanismo de valoración y defensa contra incendios. No sólo en esta ciudad se vislumbra este problema; lo es también en otras regiones y comunas de Chile, tales como Quilpué, Villa Alemana, San Antonio, Lota, Tomé, Coronel, Hualqui, Mulchén y Collipulli, Comunas en donde también existe un problema latente y potencialmente grave en futuros incendios de interfaz que podrían ocurrir. En todas ellas existe la urgente necesidad de disponer de una herramienta de gestión territorial que permita garantizar la seguridad de la población civil frente a los incendios de interfaz.
Organizaciones para la Protección Contra Incendios Forestales

\section{Corporación Nacional Forestal (CONAF)}

Desde su creación, en 1972, CONAF ha cumplido el rol de organismo técnico en protección contra incendios forestales. Le corresponde programar y ejecutar acciones que propendan a la protección de los recursos forestales, de acuerdo a lo establecido por la legislación vigente. CONAF administra un Programa Nacional de Protección Contra Incendios Forestales, mediante la organización y funcionamiento de programas regionales mediante los cuales se llevan a cabo las principales acciones de prevención y combate bajo un régimen descentralizado y de alta autonomía.

Bajo su responsabilidad, le ha tocado coordinar innumerables incendios de interfaz de distintas magnitudes y escalas de complejidad, destacándose por la alta eficiencia en la asignación de tareas y protocolos de acción para hacer frente a complicados incendios. Los casos más destacados en Chile se concentran en las regiones de Valparaíso, Bío Bío y la Araucanía, todas caracterizadas por una alta ocurrencia de incendios de interfaz. A través de acuerdos de colaboración recíproca e instancias de coordinación, se llevan a efecto diversas acciones conjuntas tales como campañas de prevención, detección y operaciones de combate.

En forma adicional, los programas regionales de Protección de CONAF están coordinados con la Oficina Nacional de Emergencia del Ministerio del Interior (ONEMI), a través de la ejecución programada de planes de contingencia para la atención de emergencias nacionales.

\section{Oficina Nacional de Emergencia (ONEMI)}

Le corresponde canalizar los esfuerzos del Gobierno, a través del Ministerio del Interior, tanto en el ámbito nacional como regional, destinados a planificar y coordinar el empleo de recursos públicos con el fin de prevenir y atender las emergencias y desastres de origen natural o antrópico. Desde 1993, ONEMI aplica protocolos específicos para las situaciones de emergencia, basada en los principios de Ayuda Mutua y Uso Escalonado de Recursos, que sustentan al Sistema de Protección Civil. Destaca el Plan de Coordinación Operativa para Situaciones de Emergencia Forestal, y que esencialmente está referido a la prevención y combate de incendios forestales de magnitud, los que por su extensión o valores afectados, amenacen la vida, salud o bienes de las personas, centros poblados, obras públicas, bosques y áreas silvestres protegidas. Las Direcciones Regionales de ONEMI, con el apoyo de las autoridades de Gobierno Interior convocan a las diversas organizaciones públicas 
que pueden colaborar en el control de las emergencias que se presentan, que incluyen a Carabineros, Fuerzas Armadas, Obras Públicas, Salud, entre otras, como así también aquellas otros servicios de utilidad pública como Cuerpos de Bomberos, Cruz Roja y Defensa Civil.

\section{Policía Forestal de Carabineros}

Es el organismo encargado de la investigación de causas de incendios y la fiscalización del cumplimiento de la legislación sobre incendios forestales y uso del fuego en zonas rurales. Le compete activa participación en situaciones donde la investigación en terreno resulta clave para dirimir y establecer eventuales responsables en la propagación del fuego. Existen casos en donde la actuación de la policía forestal ha aportado antecedentes respecto al origen del incendio y sus características originales en la propagación del fuego. La investigación de causas es clave para establecer eventuales responsables y apoyar a los procesos legales de identificación de culpables en una causa policial. En el caso de incendios de interfaz, la Policía Forestal ha permitido esclarecer variados casos de hechos intencionados que terminaron con el desarrollo de un grave incendio en la zona urbano-rural.

\section{Compañías de Bomberos}

Los bomberos han cumplido históricamente una importante labor en el combate de incendios forestales en sectores peri-urbanos de grandes ciudades, o de zonas rurales donde existe un alto riesgo a centros poblados. En el pasado incendio de Valparaíso (abril de 2014) desempeñaron una labor fundamental para que los daños producidos por la combustión masiva de casas, no hubiesen sido aún mayores. En Chile, las compañías de bomberos están integradas por voluntarios que incluso aportan cuotas personales en dinero para colaborar con su autofinanciamiento, porque la asignación que éstas organizaciones reciben desde el Estado es muy baja. Aún así, el cuerpo de bomberos de distintas compañías municipales destaca por su alto grado de compromiso con la protección civil, y se integran comúnmente a los operativos de extinción de incendios con otros organismos tales con CONAF, Carabineros, Fuerzas Armadas y voluntarios de otras instituciones de protección. En el contexto de la gestión institucional, las Compañías de Bomberos se encuentran consideradas transversalmente en todos los protocolos de protección y atención de emergencias en eventos de incendios de interfaz.

\section{Otras Organizaciones Públicas y Privadas}

Chile dispone de otras organizaciones que apoyan activamente a los sistemas de protección civil contra incendios de interfaz, y en general, en incendios forestales. Los planes de emergencias normalmente son atendidos directamente por ONEMI, quien se encarga de coordinar las acciones y tareas de Fuerzas Armadas, Cruz Roja, Defensa Civil, Servicios Meteorológicos, Servicios de Salud, Clubes aéreos y el apoyo de los servicios públicos tales como electricidad y agua potable. En casos de alerta roja activada por incendios catastróficos, la condición de riesgo a la población civil se enfrenta mediante el funcionamiento de protocolos de intervención en forma gradual y progresiva, dependiendo de las demandas zonales y la disponibilidad técnica de recursos de extinción y presupuestos de emergencia. En el caso de las faenas de evacuación, también de reconstrucción y alertas sanitarias, se activan estas organizaciones para apoyar a las instituciones principales antes mencionadas. Este apoyo se verifica tanto a nivel nacional, en lo que respecta a la formulación de políticas, estrategias globales y en el diseño e implementación de acciones técnicas específicas, como también en el ámbito regional, en el que mayormente está referido a acciones específicas de prevención y combate.

\section{La planificación territorial: un complemento necesario}

Chile presenta un alto estándar en cuanto a niveles de organización y atención de emergencias, incluso valorado a nivel internacional. Aún así, persisten serias deficiencias en aspectos de planificación territorial, labor que compete a las autoridades regionales encargadas de la gestión de espacios habitados en zonas de interfaz, particularmente los Municipios y Gobiernos Regionales. Dicho de otra forma, los esfuerzos en la gestión institucional que acá se han explicado, deben ir necesariamente coordinados con las actividades de distribución del espacio habitable mediante una adecuada planificación en Prioridades de Protección. Este aspecto se considera como una de las principales deficiencias, y en la cual existe mucha tarea por realizar. Como factor de riesgo permanente, se describen a continuación las principales referencias que debieran orientar una mejor gestión del riesgo de incendios.

Actualmente el país se enfrenta a una nueva temporada de incendios 2014-2015 con el desafío de incorporar mejoras al actual sistema de prevención y coordinación de tareas para el combate de incendios de interfaz, en atención a la fatal experiencia sucedida el pasado 14 de abril en Valparaíso. Por lo anterior, se considera que las instituciones del Estado acá descritas, han realizado sus acciones en concordancia con los actuales niveles presupuestarios, marco legal de acción y competencias profesionales que han puesto al servicio de la protección civil. Aún cuando en este ámbito también será necesario incorporar mejoras e innovación en conocimientos y pautas de acción, el problema de la protección y gestión del riesgo debe ir en función de las actuales políticas que se están aplicando al ordenamiento del espacio habitable. 
Existen graves falencias en los planes comunales de ordenación, ya que la expansión inorgánica de la interfaz crece a mayor velocidad que la infraestructura de servicios básicos. Adicionalmente existe un problema de densidad poblacional que tampoco se ha contemplado dentro de los planes de expansión y carga potencial del suelo habitable. El tema sobre el tipo de viviendas a construir y el manejo de la vegetación combustible, sin lugar a dudas que aportarán a mejorar el entorno, pero son medidas que están lejos de ser la solución al problema de fondo.

Adicionalmente existen claras evidencias del aumento en la severidad del comportamiento del fuego y un incremento de la piromanía; junto a debilidades en el actual sistema legal de sanciones. Es urgente diseñar planes de preataque y de coordinación de tareas con los medios de combate aéreos y terrestres e intentar minimizar aún más los tiempos de respuesta en el arribo de los medios de extinción. Sin embargo, la precariedad de la red de caminos y la dificultad de acceso de camiones aljibe a los sectores amagados por el fuego atenta contra las mejoras. En forma paralela es necesario efectuar una revisión exhaustiva del estado de la vegetación de propietarios privados, por cuanto a través de esos terrenos se desarrollan muchos vectores de propagación del fuego. La erradicación de casas en muchas áreas de comprobado peligro es necesaria, y aunque sea una medida dolorosa y políticamente incorrecta, es el camino a seguir.

Para finalizar, es necesario señalar que estas medidas no competen necesariamente a la gestión institucional de los organismos de defensa acá descritos, pues ellos se encargan de la protección de la población que ya se encuentra instalada en zonas de riesgo. Aún así, siempre será necesario disponer de todos los antecedentes respecto a la localización y niveles de vulnerabilidad de la población sujeta al peligro en la propagación del fuego, de manera que las acciones a ejecutar por parte de CONAF, ONEMI y organismos asociados sea aprovechada de la mejor forma posible.

\section{Comentarios finales}

Chile posee una institucionalidad desarrollada y reconocida por otros países de Sudamérica en materia de protección contra incendios forestales. El accionar de las empresas forestales y el Estado del Chile permite inferir que existe una base sólida sobre la cual se diseñan y proponen nuevas estrategias para la prevención y combate de incendios. Aún así, se considera que la evolución en investigación y desarrollo ha sido insuficiente como para poder cubrir razonablemente los aspectos más relevantes y prioritarios en materia de prevención, particularmente en eventos de interfaz. Actualmente existen iniciativas gubernamentales que permiten augurar una mayor consolidación en las normas y ordenanzas territoriales que permiten establecer donde construir, los alcances técnicos y también los aspectos básicos necesarios para educar y concientizar a la población que vive en zonas de alto riesgo de incendios. Las experiencias de graves incendios en distintas regiones de Chile permiten disponer de antecedentes suficientes para diseñar nuevas estrategias de prevención y también de combate oportuno de aquellos focos de iniciación de fuegos con propagación potencial violenta del fuego. En esta línea de acción se encuentra trabajando principalmente el Laboratorio de Incendios Forestales de la Universidad de Chile, el cual ha tenido a su cargo diversos proyectos sobre estrategias de protección civil contra incendios de interfaz urbano-forestal. El caso más concreto fue en 2010 con la ejecución del proyecto SEVEIF (Rodríguez y Silva et al., 2010), en el cual se elaboró un modelo geográfico de vulnerabilidad del espacio de interfaz, que permitió, entre otros antecedentes nuevos, poder estimar con mayor precisión el daño potencial en casas, energía, bienes y servicios y otros aspectos sociales que se encuentran en permanente peligro frente a la iniciación de un foco en áreas de interfaz. Los resultados derivados de este proyecto y otros que se ejecutaron posteriormente a ese año, permiten contar hoy con un sistema integrado de diagnóstico de peligro de incendios, que se encuentra a disposición de las autoridades de cada región, como herramienta de gestión y planificación territorial.

\section{Conclusiones}

En Chile existen instituciones, procedimientos y normas claramente establecidas para enfrentar incendios de interfaz urbano-forestal en todas las regiones del país. Es posible verificar que existe una adecuada coordinación de los distintos organismos públicos y privados en el momento de actuar frente a emergencias de gran magnitud.

Uno de los aspectos deficitarios al problema de los incendios está relacionado al incremento sostenido de la intencionalidad de este tipo de incendios, especialmente en la región Central de Chile. Aún cuando se han efectuado importantes inversiones y esfuerzos en tratar de disminuir la ocurrencia por este tipo de actividades, aparentemente la evolución y crecimiento de áreas densamente pobladas está superando con creces los esfuerzos para tratar de disminuir el riesgo de incendios en zonas de interfaz. Esta situación no es algo nueva, pues es muy frecuente también en otros países incluso más desarrollados en materia forestal y particularmente en defensa civil.

Existe claramente un déficit en investigación aplicada. Falta el desarrollo de estrategias y programas de prevención más efectivos y directos, como también 
en la implementación de programas conjuntos entre las distintas áreas de defensa del Estado, que se enfrentan a la falta de recursos financieros para dotar adecuadamente los programas de manejo del fuego en las regiones con mayor severidad de incendios.

En otras áreas del conocimiento de incendios de interfaz es posible indicar que existen importantes avances, especialmente en lo referido a la simulación del comportamiento del fuego y los niveles de severidad potenciales como elementos de pronóstico útiles para la toma de decisiones.

\section{Bibliografía}

Beebe, G., Omi, P (1993). Wildland burning: the perception of risk. Journal of Forestry, 91(9), p.19-24.

Brink, A., Mueller, C., Kilnipen, P., Hupa, M. (2000). Modeling nitrogen chemistry in the freeboard on biomass-FBC. Combust Flame 123, p. 275.

Castillo, M. (2013). Integración de variables y criterios territoriales como apoyo a la protección contra incendios forestales. Area piloto: Valparaíso Chile Central (Tesis Doctoral). Universidad de Córdoba, p. 267.

Cohen, J. D. (2000). Preventing disaster: home ignitability in the wildland-urban interface. Journal of Forestry, 98(3), p. 15-21.

Davis, J. B. (1990). The wildland-urban interface: paradise or battleground?. Journal of Forestry, 88(1), p. 26-31.

Julio, G. (2014). Fundamentos del Manejo del Fuego. VI Edición. Publicación del Depto. Gestión Forestal y Medio Ambiente. Santiago, Chile, Facultad de Ciencias Forestales y Conservación de la Naturaleza, Universidad de Chile p. 326.
KundelL, J., Myszewski, M., de Meo, T. (2002). Land use planning and policy issues. In: Hermansen, L. A., Macie, E. A. Human influences on forest ecosystems: the southern wildland-urban interface assessment. Asheville, NC: U.S. Department of Agriculture, Forest Service, Southern Research Station, p. 55-58.

Porterie, B., Consalvl, J-L., Loraud, J-C., Giroud, F., Picard, C. (2007). Dynamics of wildland fires and their impact on structures. Combustion and Flame, 149, p. 314-328.

Pyne, S., Andrews, P., Laven, R (1996). Introduction to wildland fire. 2nd ed., NY: John Wiley \& Sons, p. 808.

Radeloff, V. C., Hammer, R. B., Stewart, S. I., Fried, J. S., Holcomb, S. S., \& McKeefry, J. F. (2005). The wildland-urban interface in the United States. Ecological applications, 15(3), 799-805.

Rodríguez y Silva, F., Julio, G., Castillo, M., Molina, J-R., Herrera, M., Toral, M., Cerda, C., González, L. (2010). Aplicación y adaptación del Modelo SEVEIF para la evaluación socioeconómica del impacto de incendios forestales en la Provincia de Valparaíso, Chile. Agencia Española de Cooperación Internacional para el Desarrollo (AECID). 52p. ISBN 978-84-693-0740-3.

Stewart, S., Radeloff, W., Hammer, R., Hawbaker, T. (2007). Defining the Wildland-Urban Interface. Journal of Forestry. 105.4 (2007): 201-207.

Zhai, Y., Munn, I., Evans, D. (2003). Modeling forest fire probabilities in the south central United States using FIA data. Southern Journal of Applied Forestry $27(1)$, p. 11-17. 

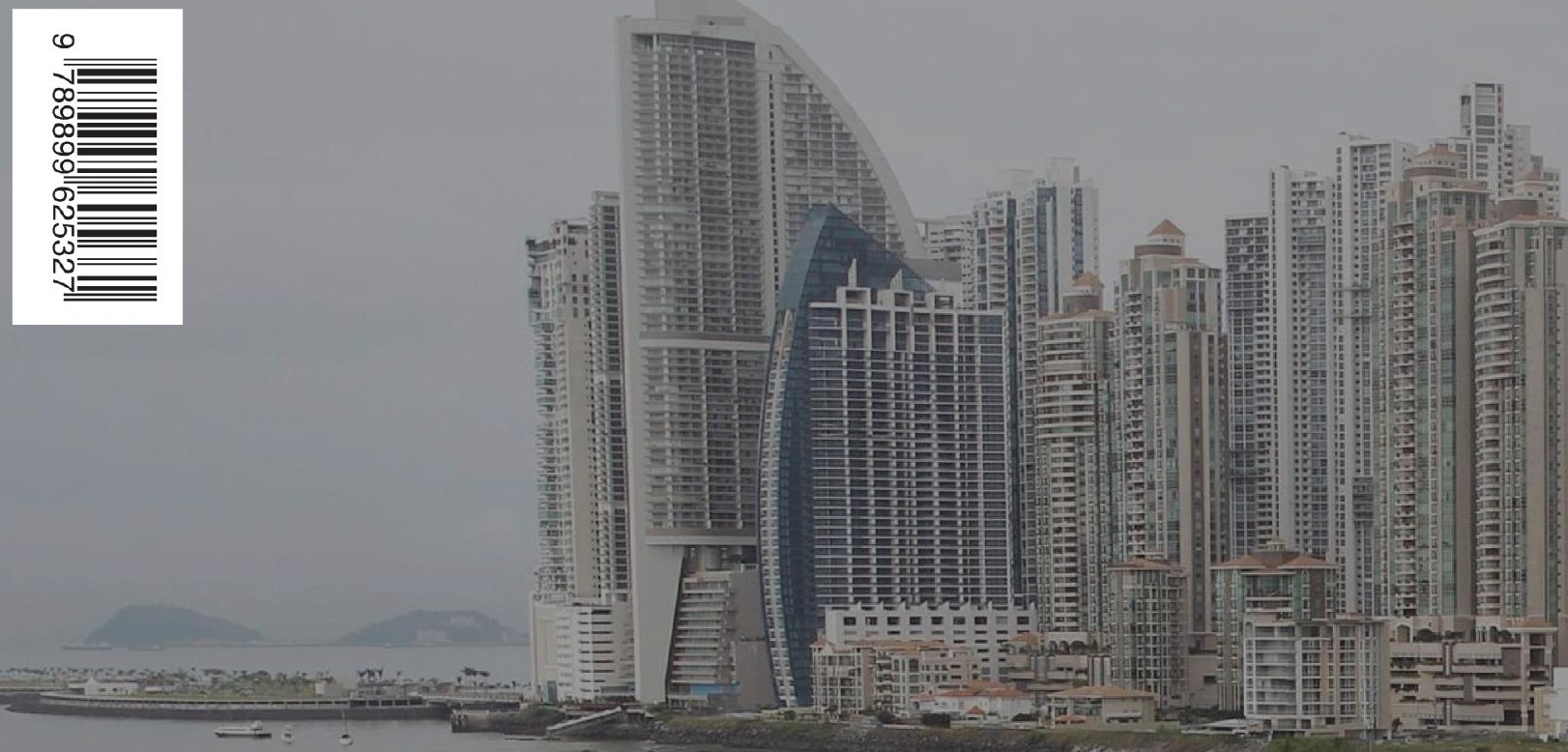

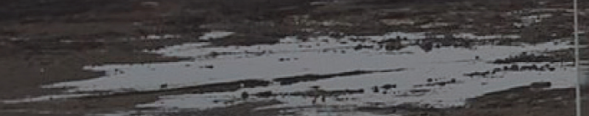

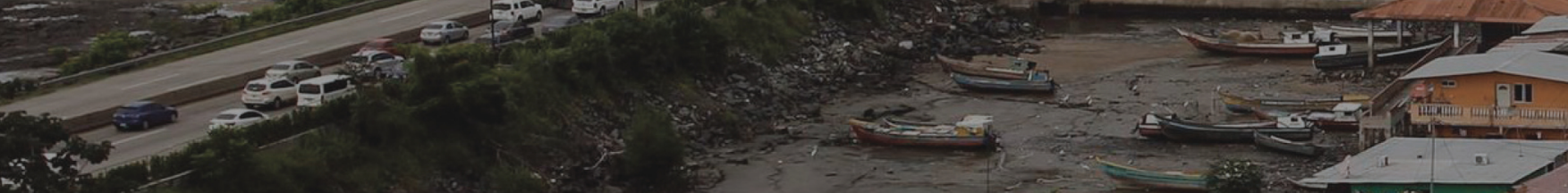

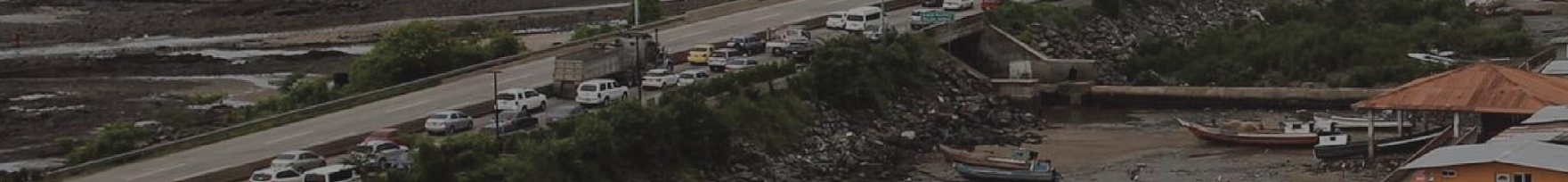
inse- $n=$ Riscos I I I

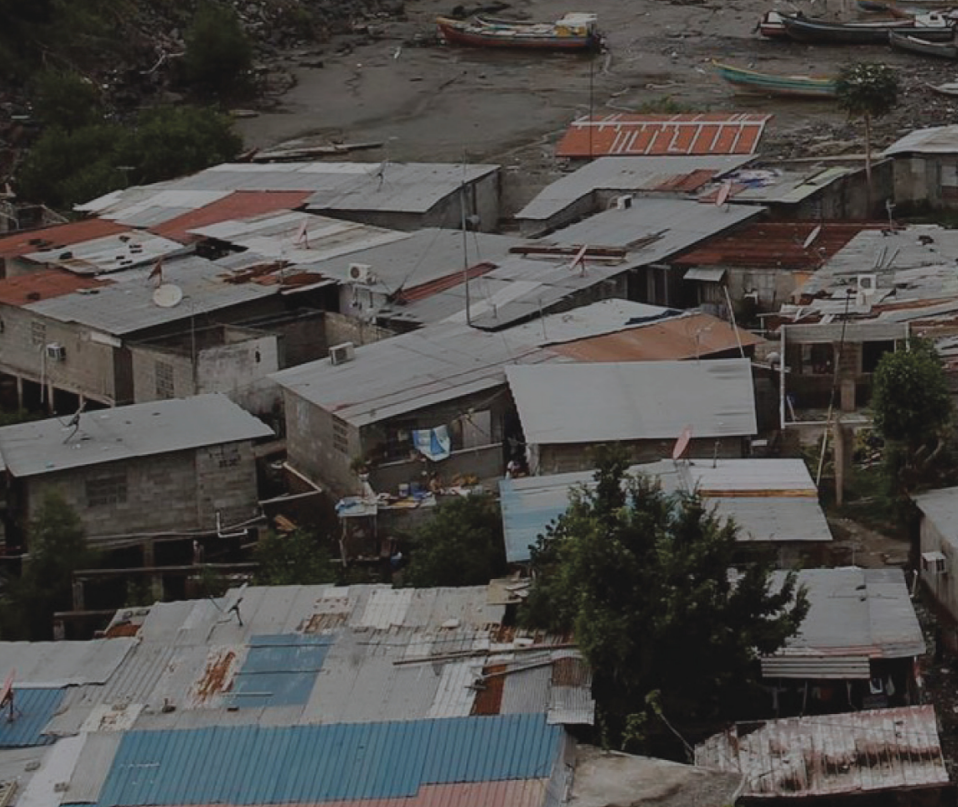

